\title{
Reliability of Knee Flexion-Extension Lyapunov Exponent in People With and Without Anterior Cruciate Ligament Deficiency
}

\author{
Salman Nazary-Moghadam, Mahyar Salavati, Ali Esteki, Behnam Akhbari, \\ Sohrab Keyhani, and Afsaneh Zeinalzadeh
}

\begin{abstract}
Objectives: The current study assessed the intrasession and intersession reliability of the knee flexion-extension Lyapunov exponent in patients with anterior cruciate ligament deficiency and healthy individuals. Study Design: University research laboratory. Methods: Kinematic data were collected in 14 patients with anterior cruciate ligament deficiency and 14 healthy individuals walked on a treadmill at a self-selected, low, and high speed, with and without cognitive load. The intraclass correlation coefficient, standard error of measurement, minimal metrically detectable change, and percentage of coefficient of variation were calculated to assess the reliability. Results: The knee flexion-extension Lyapunov exponent had high intrasession reliability, with intraclass correlation coefficients ranging from .83 to .98 . In addition, the intersession intraclass correlation coefficient values of these measurements ranged from .35 to .85 regardless of group, gait speed, and dual tasking. In general, relative and absolute reliability were higher in the patients with anterior cruciate ligament deficiency than in the healthy individuals. Conclusions: Although knee flexion-extension Lyapunov exponent demonstrates good intrasession reliability, its low intersession reliability indicates that changes of these measurements between different days should be interpreted with caution.
\end{abstract}

Keywords: gait/locomotion, motion analysis, kinematics, variability

Anterior cruciate ligament deficiency (ACLD) is a common sports-related injury commonly followed by knee instability. ${ }^{1}$. Previous studies have shown that mechanical and functional instability of the knee, impaired muscle recruitment, loss of force, muscle atrophy, and impaired neuromuscular function may affect postural control ${ }^{2}$ and gait patterns in patients with ACLD. Decreased gait variability is one of the features of gait dysfunction in patients with ACLD. ${ }^{3}$

The effect of ACLD on gait stability has been examined in relation to gait speed and dual tasking (DT). It has been suggested that slower walking speed improves gait stability. ${ }^{4}$ This assumption has been tested in different populations with balance disorders, including patients with ACLD, by examining the effect of gait speed on gait stability. ${ }^{5}$ Gait measurement under single-task (ST) conditions may limit the validity of the research. An individual's attention is usually shared between gait and other concurrent tasks. The DT phenomenon is becoming progressively popular, as people most often combine different cognitive tasks with motor tasks, such as walking or driving. These studies suggest that gait stability might be compromised during dual tasking, which would reduce balance control during walking. ${ }^{6}$

Gait variability/stability has been quantified using different measures. Even though measures derived from linear statistics,

Nazary-Moghadam and Zeinalzadeh are with the Department of Physiotherapy, School of Paramedical Sciences, Mashhad University of Medical Sciences, Mashhad, Iran. Salavati and Akhbari are with the Department of Physiotherapy, University of Social Welfare and Rehabilitation Sciences, Tehran, Iran. Esteki is with the Department of Biomedical Engineering and Physics, School of Medicine, Shaheed Beheshti University of Medical Sciences, Tehran, Iran. Keyhani is with the Akhtar Orthopedic Hospital, Shaheed Beheshti University of Medical Sciences, Tehran, Iran. Nazary-Moghadam (Nazary_salman@yahoo.com) is corresponding author. such as SD or coefficient of variation (CV), are frequently used to describe gait variability, they can only estimate the quantity of variability. In contrast, measures derived from nonlinear dynamics provide information about the temporal structure of gait variability or the manner in which gait evolves over time. ${ }^{5}$ The Lyapunov exponent (LyE) is a measure that calculates the variability during movement with inherent periodicity, and it is primarily effective for the gait analysis. ${ }^{7}$ The LyE has been examined in treadmill walking with and without cognitive loads. ${ }^{5}$ LyE measures gait stability or the sensitivity of a dynamic system to exceedingly small perturbations.

To the best knowledge of the authors, no study has been conducted to examine the reliability of the knee flexion-extension LyE in patients with ACLD and healthy subjects. It is well known that the reliability is a population- and condition-specific property. Therefore, it is necessary to establish the reliability of LyE in various musculoskeletal disorders, including ACLD, and in different experimental conditions. The purpose of the present study was to investigate the reliability of knee flexion-extension LyE at 3 levels of gait speed and at 2 levels of cognitive loading in both patients with ACLD and healthy adults.

\section{Methods}

Fourteen patients suffering from ACLD and 14 healthy individuals participated in the present study. The participants were recruited from Akhtar Hospital of Shahid Beheshti University of Medical Sciences, Tehran, Iran. Before being tested, all participants completed a questionnaire requesting demographic and injury information. The subjects with ACLD were matched to the control group according to age, sex, weight, height, walking speed, and sport activity level. The sport activity level was assessed using the Persian version of the TEGNER sport activity level questionnaire. 
All participants with ACLD had unilateral, nonacute, and complete ACL tears with and without meniscus injury. The average time since injury was 19.7 months (range 6-34 mo). Exclusion criteria for all participants were any type of serious neurological or musculoskeletal complaints or significant visual/auditory deficiencies, orthopedic injuries, as well as surgeries, medications, or medical problems that might affect balance. Before participation in this study, instructions for all testing procedures were clearly defined; each participant was asked to read the information carefully and to sign a consent form. The present study was approved by the ethics committee at the University of Social Welfare and Rehabilitation Sciences.

Participants walked on a treadmill (M8000i; Stingray, Taoyuan City, Taiwan), while their knee movements were captured by a motion capture system (Vicon Motion System Ltd $^{\circledR}$, Oxford, UK). Fifteen markers were attached to bony landmarks based on the Davis algorithm. ${ }^{8}$ The markers were placed at the left and right anterior superior iliac spine, midthigh, lateral femoral epicondyles, midtibias, lateral malleoli, the second metatarsal heads, heels, and the sacrum to record the angle of the knee in the sagittal plane using a motion capture system at $100 \mathrm{~Hz}$.

Before the start of measurements in the test session, all participants were familiarized with treadmill walking for at least 6 minutes. ${ }^{9}$ Then, the preferred walking speed was determined through a protocol. The examiner gradually increased the belt speed until the participants reported that the speed was higher than preferred speed. Then, the belt speed was then slowly decreased until the participant stated that the present speed was lower than self-selected speed. This process was repeated 3 times, and the mean of the 3 "higher than the preferred" speed and 3 "lower than the preferred" speed was considered the preferred speed. In experimental walking conditions, participants walked on the treadmill at 3 different speeds including preferred walking speed, higher than the preferred walking speed (120\% preferred walking speed), and lower than the preferred walking speed ( $80 \%$ preferred walking speed). ${ }^{5}$ In the test session, participants also practiced auditory Stroop task in the sitting position.

The experiment involved test and retest sessions, 3 to 5 days apart, accomplished by the same rater in the same laboratory. Each session consisted of 3 blocks comprising 6 walking conditions (preferred walking speed/single task, preferred walking speed/dual task, higher than preferred walking speed/single task, higher than preferred walking speed/dual task, lower than preferred walking speed/single task, and lower than preferred walking speed/dual task), which were randomly presented within each block. Each trial lasted 95 seconds, with 30-second rest between trials. The rest period between blocks was 20 minutes. The LyE was calculated using the Chaos Data Analyzer (professional version 2.1). This software was developed by Julien C. Sprott from the Physics Department of Wisconsin University, Madison, WI. The LyE estimates the separation rate of infinitesimally close trajectories. The LyE for angular knee displacement time series (sagittal plane) was measured using the Wolf algorithm. ${ }^{10}$ We did not filter the knee kinematic in sagittal plane to have precise image of the variability during mentioned trials. ${ }^{11}$ The present work only examined the sagittal plane knee kinematics because kinematic measurement from the frontal and horizontal planes collected via skin markers are correlated with increased measurement error. ${ }^{12}$ The involved knee of the subjects with ACLD was only measured. The involved limb was matched to the control group through leg dominancy. The control participants were matched to the ACLD group according to leg dominancy. If the participants' dominant leg in the ACLD group was the involved leg, the dominant leg of the control participant was evaluated. The leg used to kick the ball was defined as the dominant leg.

In single-task conditions, participants were informed to only focus on the walking. In dual-task conditions, participants were asked to give priority to both walking task and an auditory Stroop task. The auditory Stroop task consisted of a series of stimuli consisting of "high" or "low" words spoken in Persian presented with either high-pitched or low-pitched voice, resulting in 2 types of stimuli, that is, congruent stimuli where the word and the vocalization pitch matched and incongruent stimuli where the word and the vocalized pitch differed. ${ }^{13}$ In each trial, 28 to 30 words were presented with a stimulus duration of $500 \mathrm{~ms}$ and an interstimulus interval of 2.5 to 3 seconds. The participants were asked to indicate the pitch of the voice as accurately and as quickly as possible. The auditory stimulus was played to ACLD and healthy individuals via headphones, and subject's vocal response was recorded using a microphone (LEM-NP 101, Taiwan).

In the current study, the reliability of knee flexion-extension LyE has been investigated within and between days. The means of 3 trial studies carried out on motor and cognitive tasks for each condition was used to determine intersession reliability. However, any systematic change of the mean between test and retest scores, known as systematic bias, was calculated using a paired sample $t$ test. The test and retest scores were measured using the means of the 3 trials. The significance level was set at .05 for all statistical tests. In addition, relative reliability was assessed using the intraclass correlation coefficient (ICC) in 2-way random models. ICC $^{1,2}$ and ICC $^{2,3}$ were used to analyze intrasession and intersession relative reliability, respectively. ${ }^{14}$ For each ICC, a 95\% confidence interval was reported to demonstrate the precision of the estimates. The relative reliability of the kinematic parameters was categorized based on the Munro's classification. To assess absolute reliability, the standard error of measurement (SEM) was calculated as the square root of the mean square error term derived from variance analysis. In order to estimate changes caused by error measurements, the minimal metrically detectable change was used and calculated as $95 \%$ confidence interval of SEM of measure $( \pm 1.96 \mathrm{SEM})$. In addition, to compare absolute reliability between different motor and cognitive performance variables $(\mathrm{SD} / \mathrm{mean} \times$ 100), the CV was used as a measuring tool. ${ }^{15}$

\section{Results}

The $P$ values indicate that there are no statistical differences based on age, height, weight, or the TEGNER sport activity level before injury between ACLD and healthy participants (Table 1). Also, the results of present study demonstrate that none of the variables show a systematic bias in any of the conditions.

The intrasession reliability of knee flexion-extension LyE for ST and DT conditions in the ACLD group ranged from .94 to .96 and .95 to .98 , respectively. The intrasession reliability of knee flexion-extension LyE for ST and DT conditions in the healthy group ranged from .83 to .90 and .83 to .95 , respectively (Table 2).

The intersession reliability of knee flexion-extension LyE for ST and DT conditions in healthy group ranged from .41 to .53 and .35 to .68 , respectively. The intersession reliability of knee flexionextension LyE for ST and DT conditions in ACLD group ranged from .59 to .73 and .67 to .85 , respectively (Table 3 ).

Therefore, irrespective of all conditions, the patients with ACLD generally had higher ICC values than the healthy matched group. Moreover, when compared with that of the healthy 
Table 1 Demographic Characteristics of Participants With ACLD and Healthy Controls

\begin{tabular}{|c|c|c|c|c|c|}
\hline \multirow[b]{2}{*}{ Variables } & \multicolumn{2}{|c|}{$\operatorname{ACLD}(n=14)$} & \multicolumn{2}{|c|}{ Healthy $(n=14)$} & \multirow[b]{2}{*}{$P$ value } \\
\hline & Mean & SD & Mean & SD & \\
\hline Age, $y$ & 27.14 & 5.99 & 24.21 & 4.00 & .14 \\
\hline Height, $\mathrm{cm}$ & 179.00 & 5.31 & 178.14 & 6.27 & .69 \\
\hline Weight, kg & 82.43 & 13.59 & 80.14 & 29.45 & .79 \\
\hline TEGNER sport activity level ${ }^{\mathrm{a}}$ & 8.29 & 1.33 & 7.29 & 1.90 & .13 \\
\hline Walking speed, $\mathrm{m} / \mathrm{s}$ & 1.00 & 0.11 & 0.93 & 0.14 & .14 \\
\hline
\end{tabular}

Abbreviation: ACLD, anterior cruciate ligament deficient. Note: $P$ values refer to statistical significance of paired $t$ tests between mean scores of test and retest.

${ }^{\mathrm{a}}$ Range of scores: 0 to 10 points.

Table 2 Intrasession Reliability of Knee Flexion-Extension Lyapunov Exponent During All Conditions of Motor and Cognitive Difficulties in Participants With ACLD and Healthy Controls

\begin{tabular}{|c|c|c|c|}
\hline Cognitive task difficulty & $\begin{array}{l}\text { High speed } \\
\text { ICC }(95 \% \mathrm{Cl})\end{array}$ & $\begin{array}{l}\text { Self-selected speed } \\
\text { ICC }(95 \% \mathrm{Cl})\end{array}$ & $\begin{array}{l}\text { Low speed } \\
\text { ICC }(95 \% \mathrm{Cl})\end{array}$ \\
\hline \multicolumn{4}{|l|}{ ACLD } \\
\hline Dual task & $.98(.94$ to .99$)$ & $.96(.90$ to .99$)$ & $.95(.85$ to .99$)$ \\
\hline Single task & $.96(.90$ to .99$)$ & $.94(.84$ to .99$)$ & $.95(.87$ to .99$)$ \\
\hline \multicolumn{4}{|l|}{ Healthy } \\
\hline Dual task & $.95(.87$ to .98$)$ & $.92(.80$ to .97$)$ & $.83(.57$ to .94$)$ \\
\hline Single task & $.90(.76$ to .97$)$ & .87 (.67 to .96$)$ & $.83(.50$ to .95$)$ \\
\hline
\end{tabular}

Abbreviations: ACLD, anterior cruciate ligament deficient; CI, confidence interval; ICC, intraclass correlation coefficient.

Table 3 Intersession Reliability of Knee Flexion-Extension Lyapunov Exponent During All Conditions of Motor and Cognitive Difficulties in Participants With ACLD and Healthy Controls

\begin{tabular}{|c|c|c|c|c|c|c|c|c|c|}
\hline \multirow[b]{2}{*}{ Group } & \multirow[b]{2}{*}{ Gait speed } & \multicolumn{4}{|c|}{ Dual task } & \multicolumn{4}{|c|}{ Single task } \\
\hline & & ICC (95\% Cl) & SEM & CV (\%) & MMDC & ICC (95\%Cl) & SEM & CV (\%) & MMDC \\
\hline \multirow[t]{3}{*}{ ACLD } & HS & $.69(.07$ to .91$)$ & 0.05 & 35.32 & 0.1 & $.73(.13$ to .92$)$ & 0.05 & 36.33 & 0.09 \\
\hline & SS & .85 (.47 to .96$)$ & 0.04 & 40.67 & 0.08 & $.72(.04$ to .92$)$ & 0.06 & 39.69 & 0.11 \\
\hline & $\mathrm{LS}$ & $.67(.07$ to .90$)$ & 0.05 & 31.02 & 0.1 & .59 (.25 to .87$)$ & 0.05 & 29.15 & 0.1 \\
\hline \multirow[t]{3}{*}{ Healthy } & HS & $.68(-.03$ to .90$)$ & 0.09 & 54.04 & 0.18 & $.53(.17$ to .85$)$ & 0.11 & 54.71 & 0.22 \\
\hline & SS & $.46(.09$ to .83$)$ & 0.16 & 68.6 & 0.31 & $.51(.20$ to .85$)$ & 0.1 & 47.64 & 0.19 \\
\hline & LS & .35 (.11 to .73$)$ & 0.13 & 45.85 & 0.25 & $.41(.28$ to .56$)$ & 0.08 & 33.97 & 0.15 \\
\hline
\end{tabular}

Abbreviations: ACLD, anterior cruciate ligament deficient; CV, coefficient of variation; HS, high speed; ICC, intraclass correlation coefficient; LS, low speed; MMDC, minimal metrically detectable change; SEM, standard error of measurement; SS, self-selected speed.

individuals, the absolute reliability observed in the ACLD group had either lower degrees of SEM or lower degrees of CV\%.

\section{Discussion}

The present findings on the intrasession reliability showed high and very high relative reliability for all ICC values. The results further demonstrated that the ICC values obtained from the test-retest reliability of the patients with ACLD were higher than those obtained from the healthy matched group. Based on Munro's classification, ICC values greater than .70 are considered to have high reliability. ${ }^{15}$

The present methodological study enables a more accurate assessment of the differences in knee flexion-extension LyE between the subjects with and without ACLD. The intrasession relative reliability of local dynamic stability (exponential divergence) in trunk motion was measured in a study completed by Kang and Dingwell. ${ }^{16}$ The authors' findings revealed that ICC values in healthy individuals were higher than .75 during 2 and 3 minutes of treadmill walking. In this study, exponential divergence was parameterized using a double-exponential function. Our results showed that intrasession ICC values in healthy participants ranged from .83 to .95 , while the intersession ICC values ranged from .35 to .68 .

The cognitive perturbations can provoke gait dynamics, but ICC, SEM, and minimal metrically detectable change values were generally consistent across ST and DT conditions. In agreement with the result of the present study, the results of Hamacher et $\mathrm{al}^{6}$ study revealed that reliability of the trunk parameter LyE was similar during normal treadmill walking and walking while texting.

The LyE is considered a useful measure in estimating the ability to withstand perturbations in human walking. Several studies have demonstrated lower levels of knee flexion-extension 
LyE in patients with ACLD compared with those of healthy individuals. Because all these studies were conducted in one session, their findings may be comparable with those of the present study. High to very high intrasession relative reliability in both the ACLD and healthy groups indicates that measurement error might be less than the variability between participants; consequently, the possibility of error type II would be limited. An important point in this study is that reliability of knee flexion-extension LyE seems to be a sufficiently reliable measurement when comparisons between the ACLD and healthy patient groups are obtained in one session.

\section{Conclusions}

The results of the current study also revealed that the reliability of patients with ACLD is higher than those obtained from healthy controls. In addition, the findings indicate that the knee flexionextension LyE is a sufficiently reliable measure when the comparisons are obtained in one session. Lower intersession reliability for the knee flexion-extension LyE indicates that changes of such measurements occurring over a few days should be interpreted with caution.

\section{References}

1. Nazary-Moghadam S, Salavati M, Esteki A, Akhbari B, Keyhani S, Zeinalzadeh A. Gait speed is more challenging than cognitive load on the stride-to-stride variability in individuals with anterior cruciate ligament deficiency. Knee. 2019;26(1):88-96. PubMed ID: 30473374 doi:10.1016/j.knee.2018.11.009

2. Shahbazi Moheb Seraj M, Sarrafzadeh J, Maroufi N, Ebrahimi Takamjani I, Ahmadi A, Negahban H. Comparison of postural balance between subgroups of nonspecific low-back pain patients based on O'Sullivan classification system and normal subjects during lifting. Arch Bone Jt Surg. 2019;7(1):52-60. PubMed ID: 30805416

3. Knoll Z, Kiss RM, Kocsis L. Gait adaptation in ACL deficient patients before and after anterior cruciate ligament reconstruction surgery. J Electromyogr Kinesiol. 2004;14(3):287-294. PubMed ID: 15094142 doi:10.1016/j.jelekin.2003.12.005

4. Dingwell JB, Cusumano JP, Sternad D, Cavanagh PR. Slower speeds in patients with diabetic neuropathy lead to improved local dynamic stability of continuous overground walking. J Biomech. 2000;33(10): 1269-1277. PubMed ID: 10899337 doi:10.1016/S0021-9290(00) 00092-0

5. Stergiou N, Moraiti C, Giakas G, Ristanis S, Georgoulis AD. The effect of the walking speed on the stability of the anterior cruciate ligament deficient knee. Clin Biomech. 2004;19(9):957-963. doi:10. 1016/j.clinbiomech.2004.06.008

6. Hamacher D, Hamacher D, Torpel A, Krowicki M, Herold F, Schega L. The reliability of local dynamic stability in walking while texting and performing an arithmetical problem. Gait Posture. 2016; 44:200-203. PubMed ID: 27004658 doi:10.1016/j.gaitpost.2015. 12.021

7. Moraiti CO, Stergiou N, Vasiliadis HS, Motsis E, Georgoulis A. Anterior cruciate ligament reconstruction results in alterations in gait variability. Gait Posture. 2010;32(2):169-175. PubMed ID: 20591671 doi:10.1016/j.gaitpost.2010.04.008

8. Davis RB III, Õunpuu S, Tyburski D, Gage JR. A gait analysis data collection and reduction technique. Human Movement Science. 1991;10(5):575-587. doi:10.1016/0167-9457(91)90046-Z

9. Matsas A, Taylor N, McBurney H. Knee joint kinematics from familiarised treadmill walking can be generalised to overground walking in young unimpaired subjects. Gait Posture. 2000;11(1): 46-53. PubMed ID: 10664485 doi:10.1016/S0966-6362(99)00048-X

10. Wolf A, Swift JB, Swinney HL, Vastano JA. Determining Lyapunov exponents from a time series. Physica D Nonlinear Phenomena. 1985;16(3):285-317. doi:10.1016/0167-2789(85)90011-9

11. Moraiti C, Stergiou N, Ristanis S, Georgoulis AD. ACL deficiency affects stride-to-stride variability as measured using nonlinear methodology. Knee Surg Sports Traumatol Arthrosc. 2007;15(12): 1406-1413. PubMed ID: 17828526 doi:10.1007/s00167-007-0373-1

12. Cappozzo A, Catani F, Leardini A, Benedetti MG, Croce UD. Position and orientation in space of bones during movement: experimental artefacts. Clin Biomech. 1996;11(2):90-100. doi:10.1016/ 0268-0033(95)00046-1

13. Zeinalzadeh A, Talebian S, Naghdi S, Salavati M, Nazary-Moghadam S, Zeynalzadeh Ghoochani B. Effects of vision and cognitive load on static postural control in subjects with and without patellofemoral pain syndrome. Physiotherapy theory and practice. 2018; 34(4):276-285. doi:10.1080/09593985.2017.1391360

14. Shrout PE, Fleiss JL. Intraclass correlations: uses in assessing rater reliability. Psychol Bull. 1979;86(2):420-428. PubMed ID: 18839484 doi:10.1037/0033-2909.86.2.420

15. Nazary-Moghadam S, Salavati M, Esteki A, Akhbari B, Keyhani S, Zeinalzadeh A. Reliability of kinematic measures in subjects with anterior cruciate ligament deficiency during dual-task walking. J Bodyw Mov Ther. 2017;21(4):852-859. PubMed ID: 29037639 doi:10.1016/j.jbmt.2017.02.004

16. Kang HG, Dingwell JB. Intra-session reliability of local dynamic stability of walking. Gait Posture. 2006;24(3):386-390. PubMed ID: 16413784 doi:10.1016/j.gaitpost.2005.11.004 